\title{
Estado del arte de la comunicación organizacional en función de la gestión del conocimiento
}

Leidy Katherine Moreno Arenas*

DOI: https://doi.org/: 10.33571/revistaluciernaga.v12n24a4

\section{Resumen}

Se presenta un estado del arte de la comunicación organizacional en función de la gestión del conocimiento, para ello se refiere a las comunidades de prácticas en los términos de Etienne Wenger, quien las define como "grupos de personas que comparten una preocupación o una pasión por algo que hacen y aprenden a hacerlo mejor, interactuando con regularidad".

Se reseñan estudios en torno a la gestión del conocimiento en las regiones, posconflicto y el uso de los medios y las tecnologías de la información y la comunicación. Se concluye que se requieren más estudios acerca de este tema así como fomentar su discusión interdisciplinar que conlleve a la construcción social además de la organizacional.

Palabras clave: Gestión del conocimiento; comunicación organizacional; comunicación interna; comunidades de práctica.

Recibido. Junio 16, $2020 \quad$ Aceptado. Junio 30, 2020

${ }^{*}$ Comunicadora de la Universidad de Antioquia. Orcid https://orcid.org/0000-0002-6862-3285 ; email: lkatherine.moreno@udea.edu.co

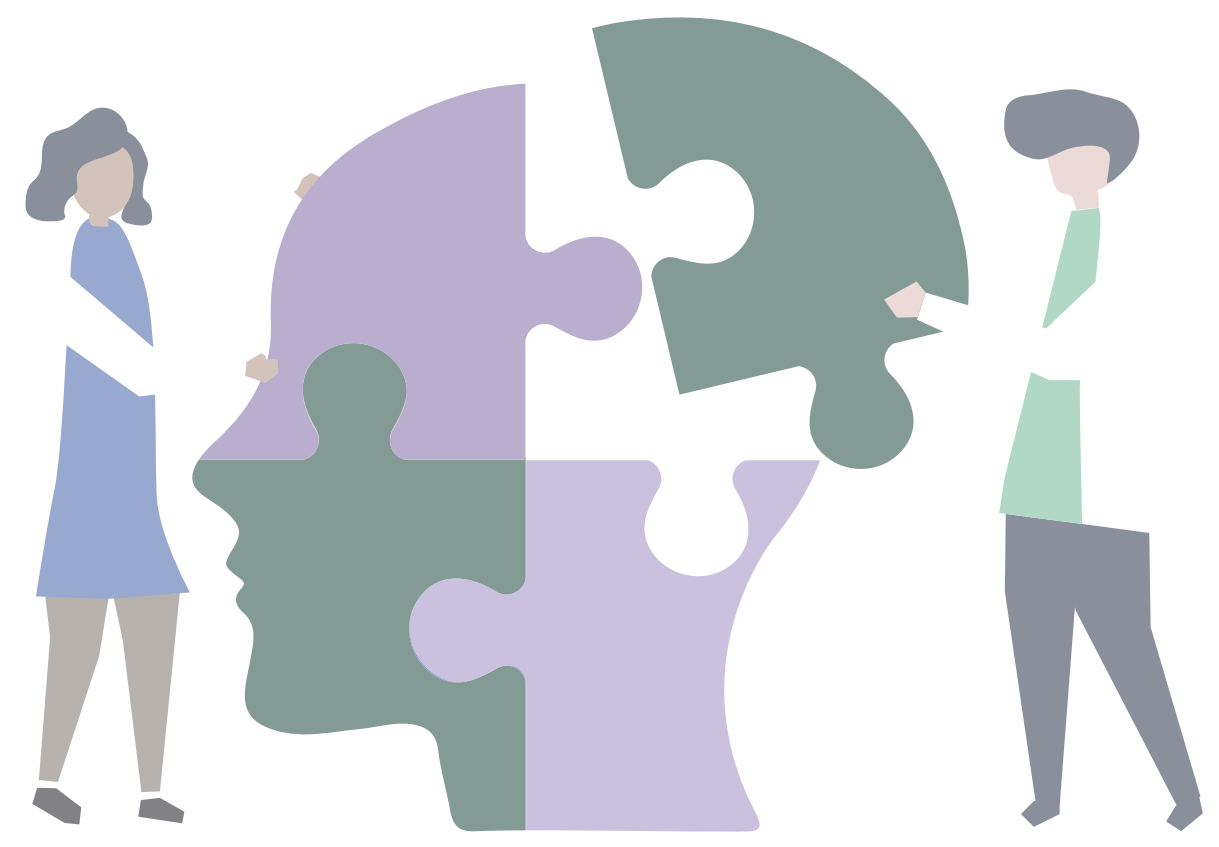




\section{State of the art of organizational communication in the light of knowledge management}

Leidy Katherine Moreno Arenas*

DOI: https://doi.org/: 10.33571/revistaluciernaga.v12n24a4

\section{Abstract}

A state of the art of organizational communication as a function of knowledge management is introduced, referring to communities of practice in the terms of Etienne Wenger, who defines them as "groups of people who share a concern or passion for something they do and learn to do it better, interacting regularly".

Studies on knowledge management in the regions, post-conflict and the use of media and information and communication technologies are reviewed. It is concluded that more studies on this topic are required, as well as the promotion of interdisciplinary discussion that leads to social and organizational construction.

Keywords:Knowledge management; organizational communication; internal communication; communities of practice.

Received. June 16, $2020 \quad$ Accepted. June 30, 2020

${ }^{*}$ Communicator of the University of Antioquia. Orcid: https://orcid.org/0000-0002-6862-3285; email: Ikatherine.moreno@udea.edu.co
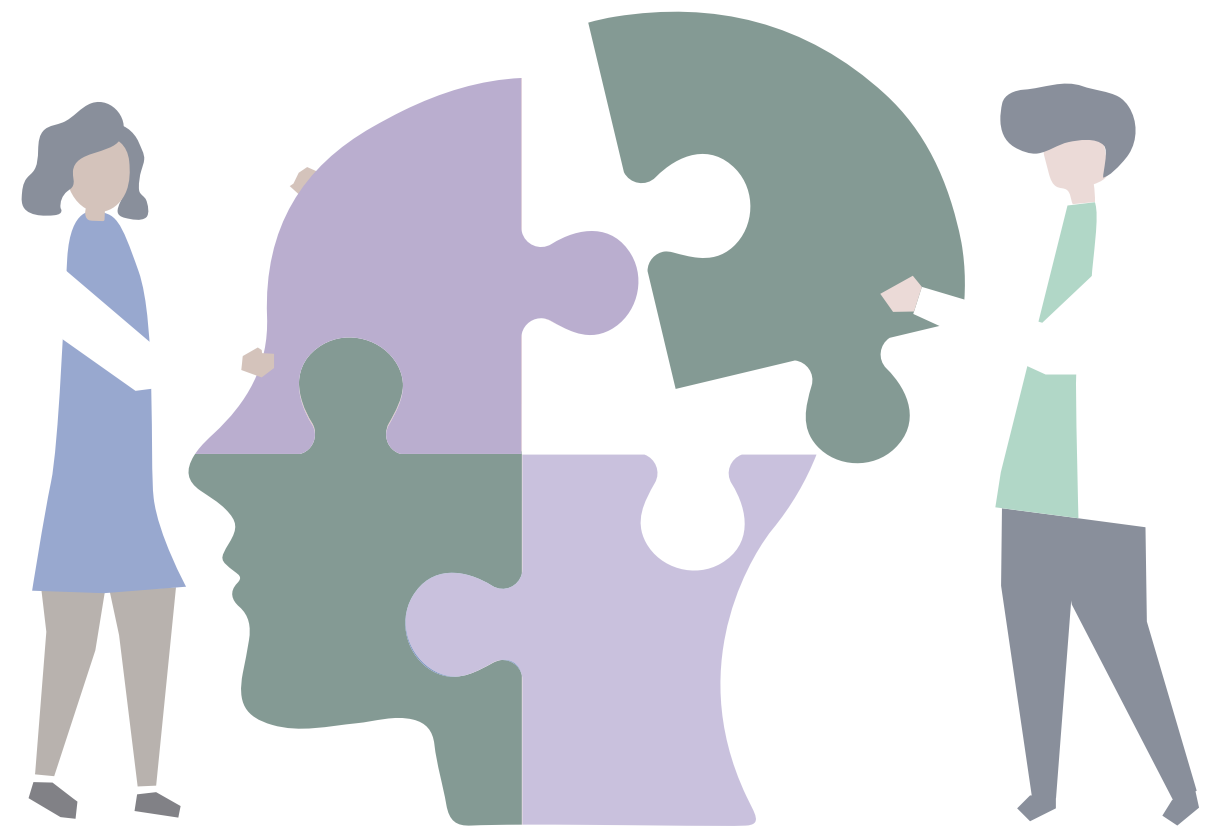


\title{
O estado da arte da comunicação organizacional em termos de gestão do conhecimento
}

Leidy Katherine Moreno Arenas*

DOI: https://doi.org/:10.33571/revistaluciernaga.v12n24a4

\begin{abstract}
É apresentado um estado da arte da comunicação organizacional em função da gestão do conhecimento, referindo-se às comunidades de práticas nos termos de Etienne Wenger, quem as define como "grupos de pessoas que compartilham uma preocupação ou paixão por algo que fazem e aprendem a fazê-lo melhor, interagindo regularmente".
\end{abstract}

Se analisam estudos sobre a gestão do conhecimento nas regiões, pós-conflito e o uso da mídia e das tecnologias de informação e comunicação. Conclui-se que são necessários mais estudos sobre este tema, bem como promover uma discussão interdisciplinar que leve à construção social e organizacional.

Palavras-chaves: Gestão do conhecimento; comunicação organizacional; comunicação interna; comunidades de prática.

Recebido: Julho 6, 2020

Aceitado: Junho 30, 2020

${ }^{*}$ Comunicadora da Universidade de Antioquia. Orcid: https://orcid.org/0000-0002-6862-3285 ; email: Ikatherine.moreno@udea.edu.co

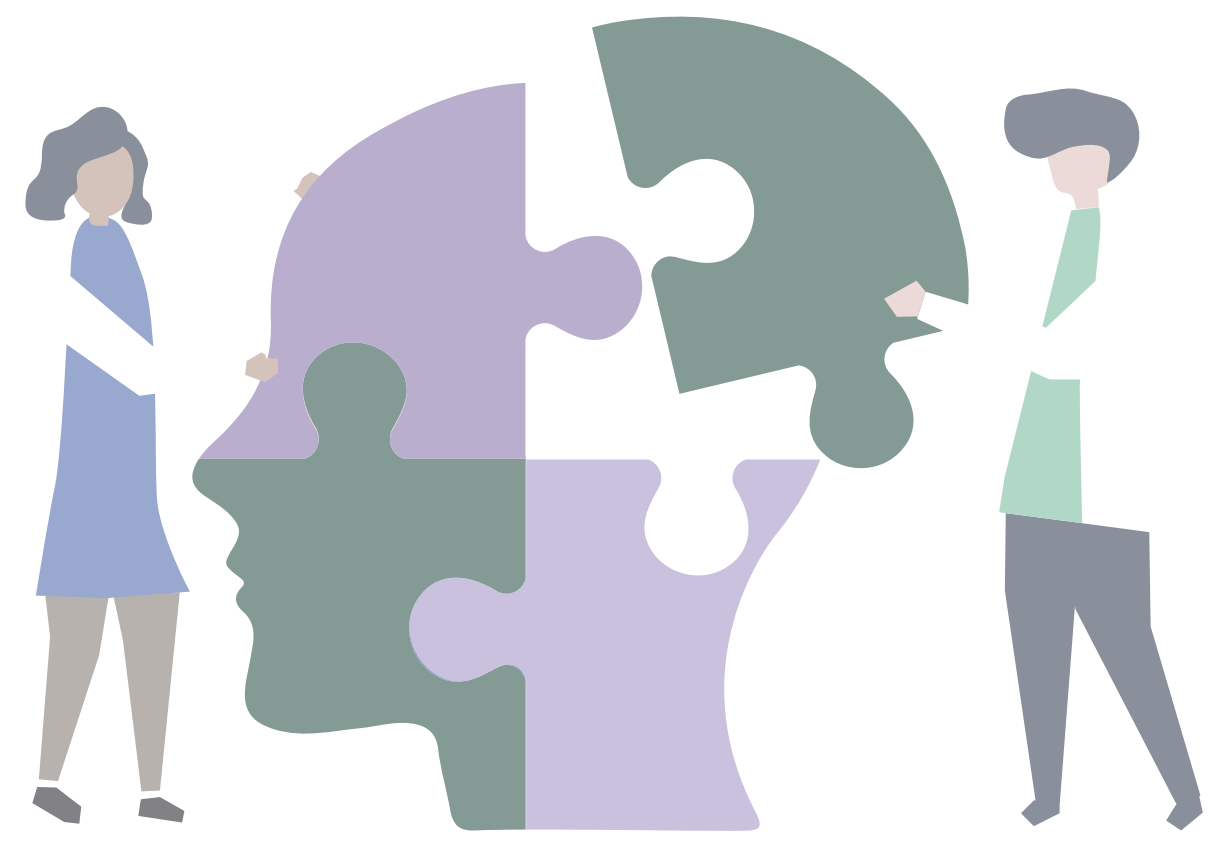




\section{Introducción}

Desde los planteamientos de Etienne Wenger (2001), la gestión del conocimiento se evidencia en las comunidades de práctica, las cuales se pueden apreciar en la vida cotidiana, escuela, trabajo y el hogar:

Las comunidades de práctica estan integradas a la vida cotidiana. Son tan informales y omnipresentes que rara vez generan interés particular, pero por las mismas razones también son muy familiares. Aunque el término puede ser nuevo, la experiencia no lo es. La mayoría de las comunidades de práctica no tienen nombre y no expiden carnés a sus miembros. Sin embargo, si durante un momento consideramos nuestra propia vida desde esta perspectiva, todos podremos construir una imagen bastante buena de las comunidades de práctica a las que pertenecemos ahora, de aquellas a las que estuvimos vinculados y de aquellas a las que nos gustaría pertenecer en el futuro (Wenger, 2001).

Estas comunidades son definidas por primera vez por Etienne Wenger, como "grupos de personas que comparten una preocupación o una pasión por algo que hacen y aprenden a hacerlo mejor, interactuando con regularidad" (Wenger, 2014). Establece a su vez, que hay tres características propias de una comunidad de práctica:

- Dominio: un interés común que conecta y se mantiene unida a la comunidad

- Comunidad: una comunidad está obligada por las actividades compartidas que persiguen (por ejemplo, reuniones, discusiones) en torno a su dominio común;

- Práctica: lo que hacen los miembros, permite su participación en la comunidad; y lo que aprenden de la comunidad afecta a lo que hacen.

Es importante mencionar que las comunidades de práctica surgen como actividad de los procesos comunicativos que implica intercambiar información y llevar a la práctica, asuntos de interés determinados por los integrantes. Las comunidades de práctica que las empresas desarrollan, buscan integrar al público a la estrategia general de la organización, a partir de la creación de flujos de acción con la finalidad de la gestión del conocimiento, la innovación y el desarrollo. Se pretende incorporar nuevos conocimientos a la empresa, y así mismo, el aprendizaje compartido.

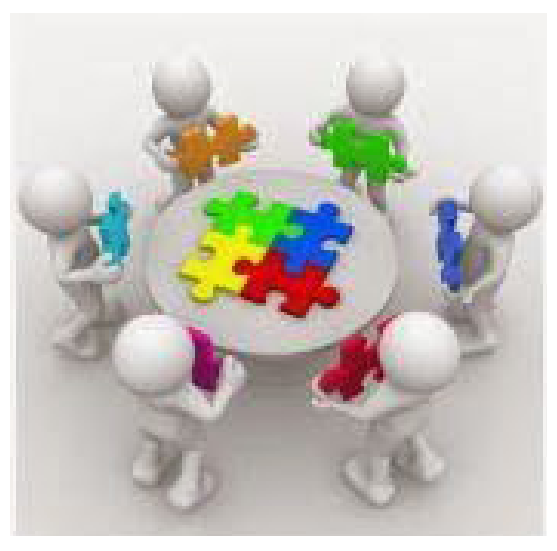


En Colombia, el grupo Nutresa y la promotora de salud Sura, cuentan con comunidades de prácticas estructuradas a partir de modelos de educación. Esta comunidades surgen ante la necesidad de las organizaciones de especializarse y de resolver retos específicos. Xerox, una empresa dedicada a la reparación de máquinas de campo, empezó a intercambiar información de interés con sus empleados sin contar oficialmente con una comunidad de práctica:

Brown y Duguid (2000) comentan que: los representantes de Xerox comenzaron a intercambiar consejos y trucos en las reuniones informales de desayuno o de almuerzo y, finalmente, Xerox detectó el valor de estas interacciones y creó el proyecto Eureka para permitir que estas fueran compartidas a través de la red mundial de representantes. Se estima que la base de datos Eureka ha permitido que la corporación ahorre 100 millones de dólares. Google y Apple también incentivan las comunidades de práctica para compartir el conocimiento entre los miembros de su personal (Bates, 2014).

Las comunidades de práctica $(\mathrm{CdP})$ han tenido mayor proyección en el área de la salud debido a sus múltiples focos o funciones de trabajo y han permitido a los profesionales de la salud intercambiar conocimiento y experiencias de las áreas afines:

Las CdP brindan un marco de colaboración para que los profesionales de la salud pública trabajen juntos para identificar y aprovechar las mejores prácticas y estándares. Mediante la colaboración en evolución y el intercambio de lecciones aprendidas en el proceso de construcción de la comunidad. (Centers for Disease Control and Prevention [CDC], 2019).

Otro ejemplo de comunidades de práctica en el área de la salud es el de la Comarca Bilbao Vizcaya, España:

La plataforma surgió ante la pregunta de cómo aprovechar toda la experiencia de las personas que trabajan en los servicios de salud, desde el personal médico hasta el administrativo, para ponerla en común y generar nuevo conocimiento. Se creó una red a través de internet, tipo blog, donde participan profesionales de los 24 centros de la Comarca Bilbao. Allí se comparte información sobre las diferentes áreas, creadas a iniciativa de los propios trabajadores, para intercambiar experiencias, recursos y enlaces en temas como el cuidado a mayores y a niños o la atención al cliente. Esta última ha resultado la más activa de todas (Ortiz, 2009).

Otro antecedente es el programa denominado "Semillas de liderazgo" dirigido por la Universidad de Antioquia. Se fundamenta en "las organizaciones que aprenden" como "un conjunto ordenado de personas [...], dentro de una atmósfera de cuestionamiento de todo lo que se piensa y se hace, y de búsqueda cotidiana de nuevas respuestas" (Universidad de Antioquia, 2001). Las CdP utilizan la comunicación para comprender los cambios y modificar los modelos mentales compartidos y comportamientos, de manera que las organizaciones puedan gestionar el conocimiento y se puedan adaptar a estos. 
Siemens plantea una ruta de trabajo desde la gestión del conocimiento en tres segmentos y secciones para que sus empleados accedan y participen:

El presidente de Siemens España, Eduardo Montes, cree que la Gestión del Conocimiento es un factor clave para convertirse en líder de innovación y contribuir así al éxito de los clientes y el desarrollo profesional de los equipos (2005).

A continuación se presenta un estado del arte de la comunicación organizacional en función de la gestión del conocimiento.

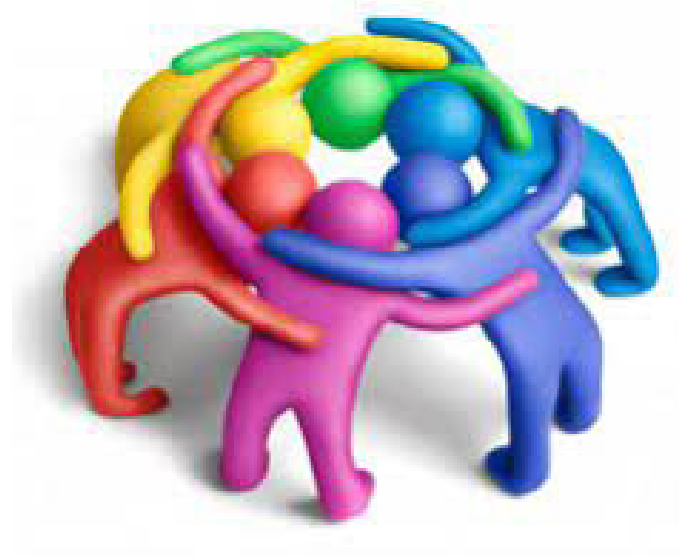

\section{Comunicación organizacional en función de la gestión del conocimiento}

La comunicación organizacional aborda el ámbito interno y externo de las empresas, actuando como formadora y transmisora de valores culturales, asumiendo a su vez, el papel transformador de la innovación en las organizaciones para la gestión del conocimiento, en el que se produce una "evolución" de la cultura y presunciones arraigadas. Se presenta como un fenómeno natural en el ámbito laboral y empresarial jugando un papel primordial en el alcance de los objetivos establecidos por la organización. Entre los actores principales se encuentran: los directivos, subdirectores, personal de recursos humanos, jefes de división, colaboradores y los clientes. Solo si existe una comunicación efectiva entre los actores, la organización podrá lograr los propósitos de una manera eficiente.

La gestión se plantea como un proceso consecuente de la comunicación, cuando es eficaz y cuenta con personal capacitado, permite el desarrollo de planes y flujos de trabajo óptimos, lo que lleva a una ejecución exitosa de los procesos empresariales.

La comunicación organizacional en función de la gestión del conocimiento se sitúa en una relación integral y holística que presenta un vínculo de doble flujo entre las personas y las empresas, en el que es tan importante el conocimiento tácito de las personas que integran la empresa como el hecho de identificarlo, almacenarlo y transferirlo por parte de las organizaciones. Es un reto para la comunicación organizacional reconocer los procesos comunicativos en función de la gestión del conocimiento para la efectividad de los procesos, así como el alcance de los objetivos misionales de las organizaciones. 


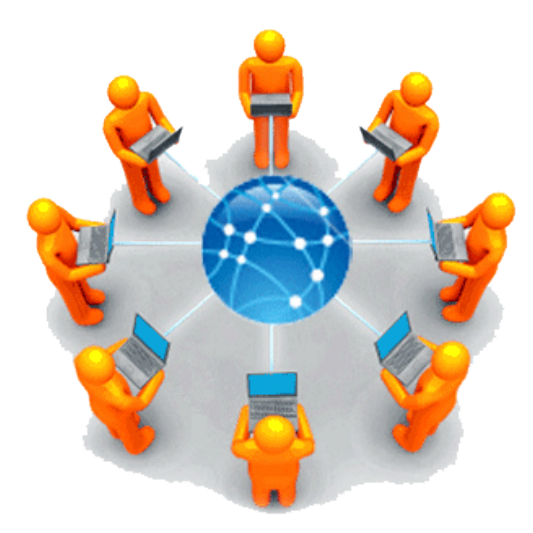

En este sentido, una de las investigaciones más recientes es una tesis de maestría sobre "La gestión del conocimiento y eficiencia en proyectos. Caso empresa Robuspack", realizada por Josué García (2019) en ella se expone la problemática de la empresa en la efectividad de proyectos, generando sobrecostos y atrasos en la producción, por lo que insisten en la necesidad de la gestión del conocimiento en la resolución de proyectos:

La investigación concluyó que la implementación de un modelo de gestión de conocimiento tiene un impacto positivo en la eficiencia de proyectos en Robuspack, al verse un incremento en la eficiencia general, de presupuesto y en tiempo de los proyectos (García, 2019).

La gestión del conocimiento regional comienza a ser explorado en Europa y Estados Unidos (Giraldo, 2017), posterior a la segunda guerra mundial como respuesta a la urgencia de la restauración, restablecimiento y funcionamiento del desarrollo industrial de las regiones. En esta línea se establece como ventaja competitiva para generar conocimiento, renovarlo y utilizarlo (Giraldo citando a Harmaakorpi y Melkas, 2005; Zhao y de Pablos, 2011, 2017).

Según PSalonius y Kapyla (2013), la Gestión de Conocimiento Regional, es entendido como "un proceso de creación en el que los actores regionales participan en la formación e implementación de una visión y estrategias regionales para promover desarrollo local, aportando su capital intelectual al desarrollo de la red" (Giraldo citando PSalonius y Kapyla, 2017). En el ambiente regional, las personas también se enmarcan en la función de colaboradores en la manera de promover el conocimiento.

Otro abordaje que se ha hecho sobre el tema es el de Quintanilla (2014), quién hace referencia a los medios y las tecnologías de la información y la comunicación en la gestión del conocimiento, no de manera instrumental, sino en la manera que guía el conocimiento, de un pensamiento concreto a uno de representación conceptual o simbólica de manera más adecuada al pensamiento.

Las organizaciones exitosas son aquellas que emplean las tecnologías de la información y comunicación para el correcto desempeño de las labores comunicativas a través de los medios más eficientes, de manera que pueda brindar a los individuos acceso oportuno a la información organizacional, que actúe en cumplimiento de los intereses empresariales. 
En este sentido en la investigación dirigida a la gestión del conocimiento en las universidades, comunicar en entornos digitales (2020), Cadena y Melgarejo (2017), plantean que "las plataformas digitales facilitan la comunicación, colaboración y la eficiencia, y tienen como objetivo impulsar la colaboración entre los principales usuarios (investigadores de las universidades, industria y gobierno) llevándolos a incrementar el impacto de la investigación. Estos autores hacen alusión a los tipos de plataformas digitales utilizadas en las universidades (académica, comercial, red social y fuente)" (Media, Almansa, \& Castillo, citando a Cadena y Melgarejo, 2020).

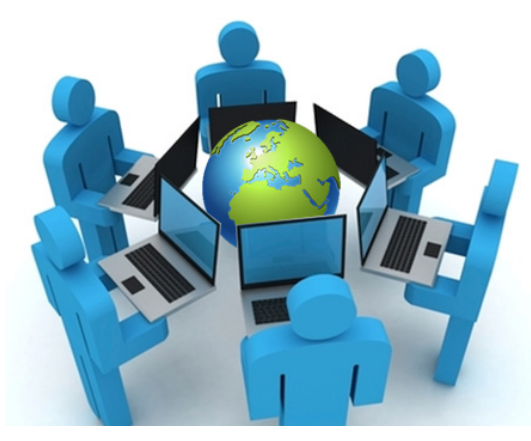

Un estudio novedoso es el de la comunicación en relación a la gestión del conocimiento en el marco del posconflicto (Patiño, 2016). Este autor invita a los receptores a fomentar el diálogo, la comunicación no violenta, el desarrollo de elementos comunicativos que promuevan la paz, y a participar de los procesos de comunicación. También hacen énfasis en la realización de procesos comunicativos dirigidos a la transmisión de saberes, a partir de micro relatos que permitan instruir a públicos que se sugieren en el posconflicto colombiano, entre ellos las corporaciones con ánimo de lucro y las instituciones gubernamentales.

En este proyecto audiovisual Patiño (2016), menciona a Luis Fernando Avendaño en el foro Comunicación, Cultura e Innovación en el Posconflicto en el que "cada vez más los medios se han convertido en una audiencia y, a su vez, en transmisores de la información”. Así en la reproducción de mensajes que puedan llegar a personas fuera del conflicto, la cultura de la paz, la conciliación y el compromiso, va a ser cada vez más consistente.

En el estudio "La gestión del conocimiento y su importancia en las organizaciones" (Goméz, D; Perez, M; Curbelo, I. 2005), se plantea a éste como un nuevo paradigma en el que existen razones tanto internas como externas que obligan a las empresas a asumir una nueva postura:

Indican los autores que Quesada y Casado, afirman: existen razones explícitas tanto internas como externas para este cambio de paradigma que obliga a las empresas a sacar el máximo provecho de su capital intelectual y por tanto de su principal intangible: sus conocimientos. Entre las razones externas: el contexto (global, virtual), los clientes (cada vez más preparados exigentes y con prioridades que cambian con mucha facilidad) y por último la competencia (recorte de los ciclos de vida de productos y servicios) (Goméz, D; Perez, M; Curbelo, I. 2005).

La cultura organizacional es trascendental para la gestión del conocimiento, independiente del carácter de la empresa, se entiende como la unión de ideas, valores, principios, 
costumbres, hábitos y tradiciones que son compartidos por todas las personas que hacen parte de una organización (Muñoz, Valencia citando a López, Marulanda e Isaza, 2015). Sin duda la comunicación hará el papel de formadora de la nueva cultura organizativa. Tal como lo propone Irene Martín (2007), la comunicación, se suscita como "un proceso de movilización y de compartir significados nuevos por medio de mensajes simbólicos" (Martín, 2007).

Lo anterior implica enfocar la cultura con el objetivo de la preparación de nuevas realidades en las que se contrarreste los procedimientos rígidos con paradigmas de flexibilidad (Muñoz, Valencia, 2015). También pretende integrar a los colaboradores en nuevos comportamientos basados en nuevos paradigmas que se afiancen en principios, valores e ideas. Por lo tanto, la comunicación es un elemento transformador importante. En este sentido, no solo las organizaciones sino también las instituciones educativas exigen una dinamización en el conocimiento como lo demuestra una investigación realizada al Instituto Universitario de Tecnología de Cabimas (Fuemayor, B; Perozo, S; Narvaez, J. 2007), en el que se encuentra la necesidad de implementar la gestión del conocimiento en las instituciones de educación superior para el desarrollo de investigaciones, tanto para aprendizaje y ampliación de conceptos y entendimientos, como en el tema de especializaciones.

Al respecto, Barrera (2003) expresa que en las instituciones universitarias las actividades de educación están determinadas por la monotonía, la falta de creatividad siendo el propósito final cumplir con unos requisitos formales, que en nada benefician el desarrollo de la investigación, como fuente auténtica del conocimiento [...] Las instituciones de educación superior no pueden quedar a espaldas de esta realidad, especialmente los institutos universitarios de tecnología los cuales por su propia naturaleza, están llamados a cumplir el rol de cambio de las funciones educativas y sin embargo, están inclinados a la repetición y copia de información, y no a la satisfacción de necesidades de su entorno intra y extrainstitucional (Fuemayor, Perozo y Narváez, 2007).

\section{Conclusiones}

A partir de este estado del arte se encuentra que la gestión del conocimiento enmarcado en la comunicación organizacional no se limita solo a la disciplina de la gestión del conocimiento, empero fomenta la discusión interdisciplinar en la formación de nuevos conocimientos y en función de la construcción social.

Se evidencian estudios en torno a la gestión del conocimiento en las regiones, el posconflicto y el uso de los medios y las tecnologías de la información y la comunicación. Se concluye que se requieren más estudios acerca de este tema así como fomentar su discusión interdisciplinar que conlleve a la construcción social además de la organizacional.

Se requieren investigaciones que hagan referencia a la gestión del conocimiento en las organizaciones que permita avanzar en dicha temática, en especial en la metodologías y acciones utilizadas en las comunidades de prácticas. 


\section{Bibliografía y Cibergrafía}

Artiles, S. (2009). La gestión documental, de información y el conocimiento en la empresa. El caso de Cuba. La Habana, Cuba. Revista SciElo. Disponible en: http://scielo.sld.cu/scielo.php?script=sci_artte $\underline{x t \& p i d=S 1024-94352009000500002}$

Bates, T. (2017). Enseñar en la Era Digital. Una guía para la enseñanza y el aprendizaje. Buenos Aires, Argentina. Universidad de Buenos Aires. Recuperado de: https://cead.pressbooks.com/ chapter/4-6-las-comunidades-de-practica/

CDC, 2019. Communities of Practices (CoPs). Center for State, Tribal, Local, and Territorial Support. Recuperado de: https://www.cdc.gov/phcommunities/

CEPAL. (2004). América Latina y el Caribe rezagada en investigación y desarrollo. Recuperado de: https://www.cepal.org/es/comunicados/america-latina-caribe-rezagada-investigacion-desarrollo

Caballero, S. (2008). La gestión del aprendizaje Algunas preguntas y respuestas sobre en relación con el desarrollo del pensamiento en los estudiantes. Revista Polis. Santiago, Chile. Tomado de: https://scielo.conicyt.cl/pdf/polis/v7n21/art15.pdf.

Canary, H. McPhee, R. (2011). Communication \& Organizational Knowledge: Contemporary Issues for Theory and Practice. Arizona State. Routledge.

Careaga, M. (2013). La Gestión del Conocimiento y los desafíos de inserción en Educación (Parte 1) Cuestiones conceptuales. Concepción, Chile. Universidad Católica Santísima de la Concepción. Recuperado de: https://www.ucsc.cl/wp-content/uploads/2013/07/GC+EDUCACION.pdf

Castro, C; Gracia, S.(2014) Metodología de Evaluación y Gestión del Conocimiento dinámico por procesos utilizando como soporte TIC el Entorno Colaborativo de Trabajo basado en el modelo de creación de Conocimiento de Nonaka-Takeuchi. Caso de estudio en el área de Gestión de proyectos de I+D+i en institución avanzada en Conocimiento. Servicio de Publicaciones de la Universidad de Córdoba. Montería, Colombia. Tomado de: https://core.ac.uk/download/pdf/60898157.pdf.

Collado, F. (1999). La comunicación en las organizaciones. México, Trillas.

Fuemayor. B; Perozo. S; Narvaéz, J. (2007) Investigación y gestión del conocimiento. Caso Instituto Universitario de Tecnología de Cabimas. Laurus. Caracas Venezuela. Tomado de: https://www. redalyc.org/pdf/761/76111485018.pdf.

Gamba, M. Montilla, M. Villarreal, S. (2019). Estado del arte de la producción investigativa en la línea de educación y medios interactivos 2011 -2018. Trabajo de grado para optar por el título de Magíster en Comunicación. Bogotá. Colombia.

Garcia, J. (2019). Gestión del conocimiento y eficiencia en proyectos. Caso empresa Robuspack. Maestría en dirección y gestión de proyectos de ingeniería. Centro de tecnología avanzada. México. Tomado de: https://ciateq.repositorioinstitucional.mx/jspui/bitstream/1020/302/1/ GarciaArzateJosue\%20MDGPI\%202019.pdf 
Giraldo, C. (2017). La gestión del conocimiento en las organizaciones y las regiones: Una revisión de la literatura. Tendencias. Nariño, Colombia. Recuperado de: https://www.redalyc.org/ pdf/695/69542291012.pdf.

Godói, E. Eiko, L. (2013). Comunidades de Práctica Una Innovación en la Gestión del Conocimiento. Journal of Technology Management \& Innovation. Santiago, Chile. Tomado: https://scielo.conicyt.cl/ pdf/jotmi/v8s1/art11.pdf.

Gómez, D; Pérez. M; Curbelo, I. (2005) Gestión del conocimiento y su importancia en las organizaciones. Ingeniería industrial. La Habana, Cuba. Tomado de: https://www.redalyc.org/ pdf/3604/360433559006.pdf.

Grau, A; Lara E; Civit, R \& Sieber, S. (2005). Comunidades de Práctica en Siemens: ¿conviene controlarlas? IESE Insight. Barcelona, España. Tomado de: https://www.ieseinsight.com/doc. aspx?id=443\&ar=10\&idioma $=1$.

Hernández, R; Pérez, M. Morales, E. (2012). Gestión del aprendizaje: referente innovador para la formación de académicos en la Universidad Veracruzana. II Congreso Internacional de Educación Superior. Del 29 al 31 de octubre 2012. Chiapas, México. Tomado de: https://www.uv.mx/ formacionacademica/files/2019/07/x4-referente-innovador.pdf.

Hoyos, C. (2000). Un modelo para investigación documental. Guía teórico-práctica sobre construcción de estados del arte con importantes reflexiones sobre la investigación. Medellín, Colombia: Señal editorial. Recuperado de: https://es.scribd.com/doc/16281901/UN-MODELO-PARA-INVESTIGACIONDOCUMENTAL-29-04-08

Martín, I. (2007). Retos de la comunicación corporativa en la sociedad del conocimiento: de la gestión de información a la creación de conocimiento organizacional. Signo Y Pensamiento. Recuperado de: https://revistas.javeriana.edu.co/index.php/signoypensamiento/article/view/4594

McAnally, L. Organista, J. (2007). La educación en línea y la capacidad de innovación y cambio de las instituciones de educación. Baja California, México. Apertura. Recuperado de: file://C:/Users/Leidy/ Downloads/McAnallyyOrganista\%20(3).pdf

Media, V; Almansa, A \& Castillo, A. (2020). Gestión del conocimiento en las Universidades. Comunicar en entornos digitales. Inmediaciones de la comunicación. Montevideo, Uruguay. Recuperado de: https://www.researchgate.net/publication/342148923 Gestion_del conocimiento en_las_universidades_Comunicar_en_entornos_digitalesKnowledge management_in_universities Communication in digital_environments

Minaka, A. (2009). Gestión del conocimiento en educación y transformación de la escuela. Notas para un campo en construcción. Sinética. Jalisco, México. Tomado de: http://www.scielo.org.mx/scielo. php?script=sci_arttext\&pid=S1665-109X2009000100008.

Ministerio de educación. (2011). Gestión del conocimietno https://www.mineducacion.gov.co/1759/ articles-324587_archivo_pdf_4_Gestion_Conocimiento_MEN.pdf 
Muñoz, D; Valencia, J. (2015). Gestión del conocimiento organizacional: un encuentro necesario entre plataformas digitales, comunicación, educación y cultura. Revista Lasallista. Medellín, Colombia. Recuperado de: https://www.redalyc.org/pdf/695/69542291012.pdf.

Nava, J. (2017). La esencia del conocimiento. El problema de la relación sujeto-objeto y sus implicaciones en la teoría educativa. Guadalajara, México: Revista SciElo. Recuperado de: http:// www.scielo.org.mx/scielo.php?script=sci_arttext\&pid=\$2007-74672017000200025

Londoño, O. Maldonado, L. Calderon, L. (2016). Guía para construir estados del arte. Bogotá, Colombia: ICONK. Recuperado de: https://iconk.org/docs/guiaea.pdf

Ortiz, A. (2009). Comarca Bilbao, un ejemplo de comunidad de práctica profesional a través de la web 2.0. Euskadinnova. Recuperado de: https://www.spri.eus/euskadinnova/es/innovacion-social/noticias/ comarca-bilbao-ejemplo-comunidad-practica-profesional-traves-20/5342.aspx

Patiño, A. (2016). Comunicación y gestión del conocimiento: Proyecto audiovisual de negociación para la compañía Grans Consulting. Trabajo de grado para optar por el título de Comunicador Social Campo Organizacional. Bogotá, Colombia. Recuperado de: https://repository.javeriana.edu.co/ bitstream/handle/10554/20106/PatinoChicuasuqueAndresFelipe2016. pdf?sequence=2\&isAllowed=y.

Pérez, A. Cruz, Y (2012). Posiciones teóricas en torno a la comunicación organizacional. EUMED. Recuperado de: https://www.eumed.net/rev/cccss/20/cbfp.html

Pérez, N. (2018). Gestión del conocimiento tácito: análisis de la disociación teórico-práctica enfermera en el bloque quirúrgico. Tesis doctoral. Alicante. Recuperado de: https://rua.ua.es/dspace/ bitstream/10045/99690/1/tesis natalia_perez fuillerat.pdf

Portilla, M. Rojas, A. y Hernandez, I. (2014). Investigación cualitativa: Una reflexión desde la educación como hecho social. Nariño, Colombia: Docencia, investigación, innovación. Recuperado de: https://revistas.udenar.edu.co/index.php/duniversitaria/index

Quintanilla, N. (2014). Herramientas y TIC 's en la gestión del conocimiento. El Salvador: Centro de Investigación y Transferencia de tecnología. Recuperado de: http://rd.udb.edu.sv:8080/jspui/ bitstream/11715/621/1/Herramientas\%20TICs\%20y\%20Gestion.pdf

Romero, R. (2013). La Comunicación Interna y el Trabajo en Equipo en la empresa Constructora Alvarado de la Ciudad de Ambato. Ambato, Ecuador: Universidad Técnica de Ambato. Recuperado de: http://repositorio.uta.edu.ec/bitstream/123456789/5698/1/80\%20o.e..pdf

Universidad de Antioquia. (2001). Organizaciones que aprenden. Recuperado de: http://huitoto.udea. edu.co/Liderazgo/practicas/lecturas/cap6/ORGANIZACIONES_QUE_APRENDEN.html

Thomas, H (2006). La importancia de la comunicación interna a través de internet. Área de recursos humanos. 17 de junio del 2006. Tomado de: http://www.yahoo.com.mx.

Valdettaro, S. (2011) McLuhan: Pliegues, trazos y escrituras-post. Editorial de la Universidad Nacional de Rosario. Bogotá, Colombia. Tomado de: https://core.ac.uk/download/pdf/61698851.pdf. 
Valle, M.; Herrera, T. (2020). Cluster Comunidade de Medellín. Do discurso da guerra ao da colaboração. DOI: 10.22533/at.ed.92821060111

Valle, M.; Herrera, T. (2019). Comunidad Clúster de Medellín. Pensar las estrategias y el estratega de la comunicación desde los sistemas abiertos. Brazilian Journal of Development (BJD). Vol. 5. Núm.7. DOI: https://doi.org/10.34117/bjdv5n7-182

Valle Flórez, M. M.(2016). Organizational Communication Research in Antioquia Colombia. Luciérnaga Comunicación, 7(13), 66-76. Recuperado a partir de https://revistas.elpoli.edu. co/index.php/luc/article/view/742

\section{Para citar este artículo}

Moreno, L. (2020). Estado del arte de la comunicación organizacional en función de la gestión del conocimiento. Revista Luciérnaga Comunicación. Vol. 12 Núm. 24. Pp.58-70. https://doi.org/: 10.33571/revistaluciernaga.v12n24a4

OJS: https://revistas.elpoli.edu.co/index.php/luc/issue/archive

Link: https://www.politecnicojic.edu.co/index.php/revista-luciernaga 